\title{
Concept and Development of Next Phase Gateway Applied to Internet of Things
}

\author{
Eisei Yoshida ${ }^{1+}$, Tetsuya Yokotani ${ }^{1}$ and Koichi Ishibashi ${ }^{2}$ \\ ${ }^{1}$ Department of Electrical and Electronics Engineering, College of Engineering, Kanazawa Institute of \\ Technology, Nonoichi, Ishikawa, Japan \\ ${ }^{2}$ Department of Information and Computer Science, College of Engineering, Kanazawa Institute of \\ Technology, Nonoichi, Ishikawa, Japan
}

\begin{abstract}
Gateway was developed as a home adapter to be used in home security and controlling household appliances. However, its requirements changed with improvements in network technologies, and they can be divided into four phases. With increasing development of Internet of Things (IoT), internet services have become more diverse in recent years. Therefore, requirements of gateways, for their application to IoT, have also changed. The requirements of a phase 5 Gateway are open connectivity and capability to cooperate with multiple cloud services. In this study, a wildlife monitoring system, which is a use case in the FED4IoT project, was chosen in the development of the Phase 5 Gateway. A data model was created to make the gateway learn the type of data obtained at the gateway. Finally, a phase 5 Gateway to be applied to the wildlife monitoring system was developed.
\end{abstract}

Keywords: IoT, Gateway, Data federation, Semantic interoperability, FED4IoT

\section{Introduction}

Gateway was developed as a home adopter to be used in home security and controlling household appliances. With improvements in network technologies, the gateway has been also improved over two decades. In this paper, we have defined the term of the gateway as a device that enables new network services realized by connecting home devices in home and/or local networks to the Internet [1].

In recent years, Internet of Things (IoT) over the $5 \mathrm{G}$ mobile system have been attracting widespread interest in ICT perspective. In addition, internet services have become more diverse and requirements of the Gateway have varied. With the establishment of the passive optical network (PON), high-quality broadband services using the optical access network can be provided [2]. Consequently, high-quality communication services have become available as next generation networks (NGN). This has led to the development of a home Gateway, which realizes multiple services, such as the telephone, video distribution, and the Internet. In recent years, a high-capacity optical access network caused by $4 \mathrm{k} / 8 \mathrm{k}$ and traffic offloading of LTE/5G has gained attention. Moreover, the transfer of enormous and minute data through the IoT is a critical issue. In these situations, the requirements of a gateway that connects the optical access network and home network have changed. In this study, we discuss the evolution of the Gateway and then present its requirements when applied to IoT. Finally, the development of the IoT gateway is summarized.

\section{Evolution of the Gateway}

\subsection{Diversification of Internet Services and Transition of Contents}

In recent years, internet services have varied with the diversity in the network equipment and the Internet content, such as broadcasting services with $4 \mathrm{k}$ or 8k [3] and IoT [4]. Therefore, it is expected that the characteristics of communications will expand into the minute data with an enormous number of communication flows. Thus, as the ultra high-definition distributions, such as the $4 \mathrm{k} / 8 \mathrm{k}$ and IoT are introduced, the relationship between the access frequency and content size is obtained as shown in Figure 1.

\footnotetext{
+ Corresponding author.

E-mail address: b1695179@ planet.kanazawa-it.ac.jp
} 
In this figure, the horizontal axis is the access frequency (occurrence frequency of traffic), and the vertical axis is the content size (amount of traffic in a single traffic). Recently, with the introduction of IoT, many governments and companies are discussing smart-city applications [5] and smart factories [6]. Thus, it is expected that the lower right zone in Figure 1 will increase in addition to the increase in the spread of the IoT. Real-time is considered necessary along with the diversity in the traffic characteristics. As shown in Figure 1, the Gateway needs to handle high-speed traffic with a few flows and low-speed traffic with enormous flows. Therefore, it should be capable of handling a scale-free traffic.

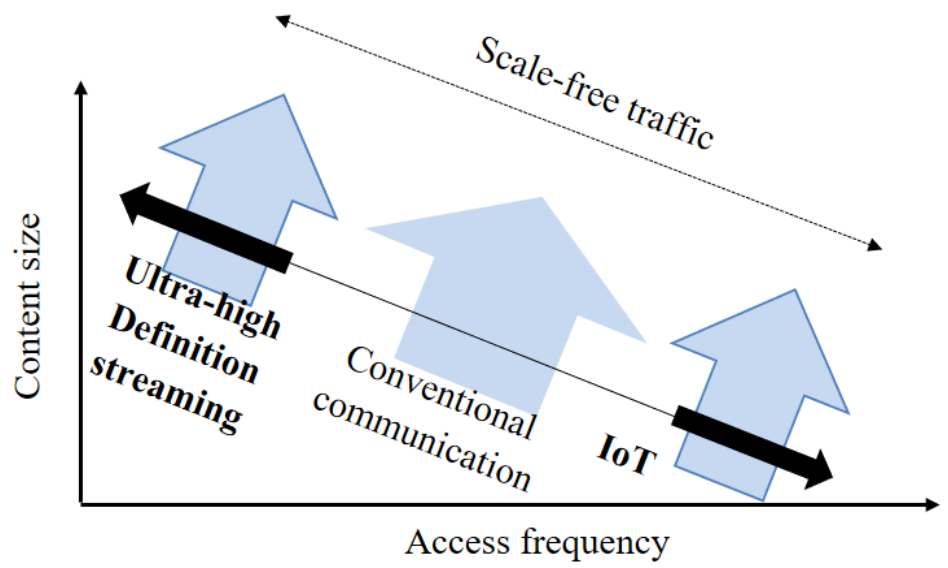

Fig. 1: Relationship between the access frequency and content size

\subsection{Requirements of the Conventional Home Gateway}

The transition in the requirements of gateways is shown in Figure 2. In this figure, the horizontal axis is multifunctional, and the vertical axis shows the performance. Phase 1-phase 4 gateways are conventional whereas the phase 5 Gateway is intended for IoT use.

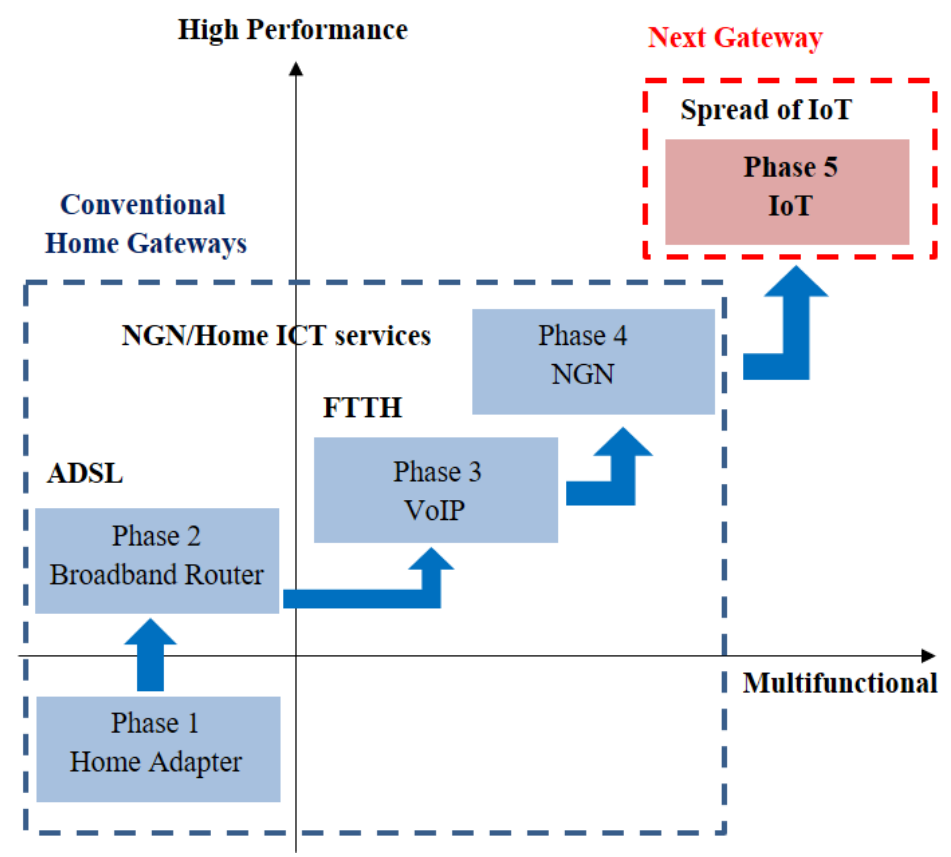

Fig. 2: Transition of the requirements of the home Gateway

1) Phase 1: home adapter

Gateway was developed for home security and controlling the household appliances. In this phase, the number of equipment connected to the Gateway was limited, and its function was specialized to providing services and applications. Because internet services were provided by the integrated services digital network (ISDN), which is low in speed and pay-per-use, wide area communication was not frequent and the gateway was primarily used for communication within a building and/or home. 
2) Phase 2: broadband rooter

With the development of the asymmetric digital subscriber line (ADSL), a fixed charge was realized. Therefore, the Internet access became the gateway's primary use, and the Gateway was transformed into a broadband router. In this phase, smooth communication with the wide area network was required for the Gateway, and a throughput of dozens of $\mathrm{Mb} / \mathrm{s}$ was required. In addition, use of several computers in a home network was assumed, and an IP process (such as NAT/NAPT and DHCP server) was required.

\section{3) Phase 3: VoIP terminal adapter}

With the development of fiber to the home (FTTH), the communication quality was remarkably improved. Therefore, high-quality voice over IP (VoIP)became popular and the Gateway had to be applied to the VoIP. At that time, only best-effort delivery was used for the Internet services, and packet length was comparatively long. However, a guarantee of real-time, which ITU-TY.1541 prescribes as $50 \mathrm{~ms}$ end to end, and capability to handle voice packet with short packet length, was required. Therefore, priority control of telephone services and traffic from the Internet access were required. To realize such a control, improvement in the processing performance of the Gateway was expected.

\section{4) Phase 4: home Gateway applied to the NGN}

With the spread of NGN, which includes all the services in IP, video distribution as a service was added to the main Internet services (telephone/the Internet access). In addition, the home Gateway had to be applied to triple services (telephone/video distribution/internet access). Consequently, the performance of the Gateway was expected to improve. In addition, a function for IPv6 (such as IPv4/IPv6 transformation and IP-SEC) was required in the Gateway because NGN is based on IPv6.

Moreover, home ICT services were required to be provided to the users. Visualization of consumed energy and observation services for home monitoring from remote areas are typical examples [7]. To realize these services, there was a need to develop a platform capable of containing the software specialized in services, which users join. The implementation of a platform based on open services Gateway initiative (OSGi) alliance [8] is a typical example of the same.

In this phase, high-quality software distribution was realized by taking advantage of the implementation of OSGi. In addition, home network management was required to provide seamless connections between NGN and home networks on an IP level in the home ICT.

\section{5) Phase 5: IoT Gateway}

In the phase 5, the Gateway supports IoT services. Especially, various physical interfaces to connect IoT devices are implemented. Moreover, this gateway supports connectivity of application data to servers. By the way, some IoT services are deployed in outside. Therefore, this gateway is installed in some outside plants in addition to inside of buildings

\section{Requirements of Phase 5 Gateway}

Phase 5 Gateway applied for IoT usage is essentially an improvement over the phase 4 Gateway. Its requirements consist of "open connectivity towards multiple services" and "cooperation with cloud services." In this section, these two requirements have been presented in detail.

\subsection{Open Connectivity Towards Multiple Applications}

With an increase in the propagation and application of IoT, sensors and other IoT devices in the home network services are connected with the phase 5 Gateway in addition to the conventional triple services (telephone/video distribution/internet). Although it seems similar to the home ICT services described in Section 2, further open connectivity is expected for IoT use. The phase 5 Gateway, which is able to connect diverse cloud services for IoT use, is shown in Figure 3. In this figure, the gateway is connected to various networks specialized in service use. 


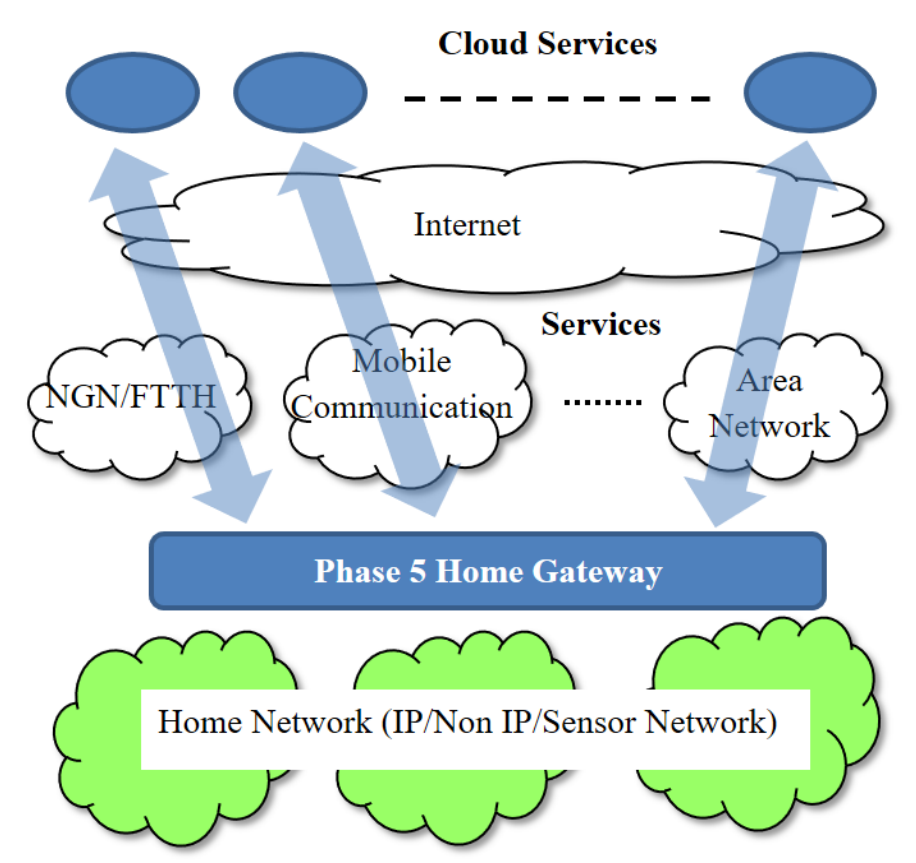

Fig. 3: Open connectivity in Phase 5 Gateway

\subsection{Cooperation with Multiple Cloud Services}

Depending on the IoT applications, real-time performance is an essential requirement. If it is not fulfilled, the traffic occurs locally. Although traffic occurring in a home network is generally gathered and processed in a cloud server, integration division and data analysis division are required considering the communication performance and efficiency of the IoT. For example, when a fire breaks out, the sensors in a home network detect it. However, the service provider needs to analyze the received data, which takes a long time. It is quicker and better to integrate, analyze, and transfer the data to the service provider. Thus, the Gateway works as an edge part of edge computing, as shown in Figure 4. Consequently, the phase 5 Gateway needs to cooperate with the cloud services as well as the communication processing.

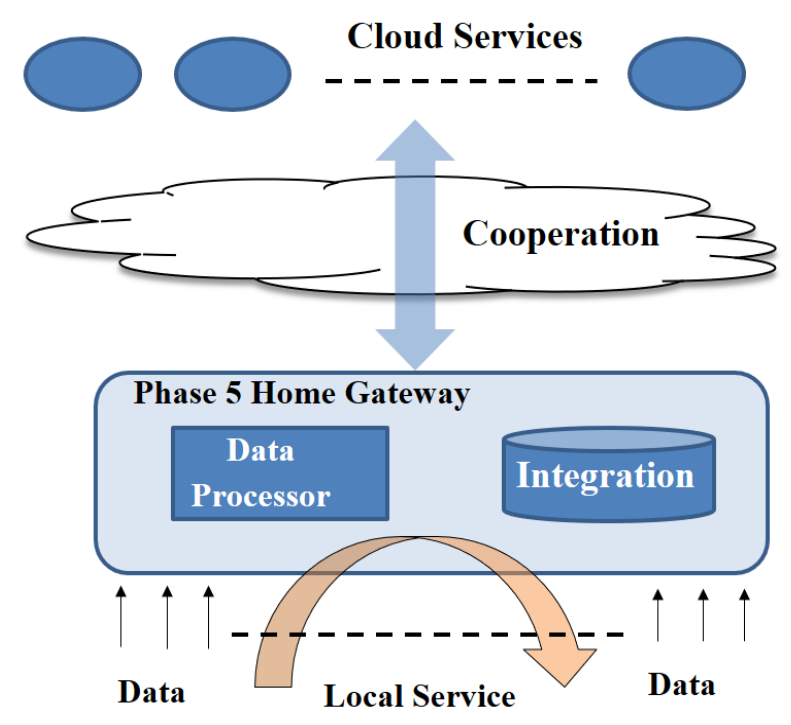

Fig. 4: Structure of phase 5 Gateway integrated with cloud services

\section{Development of Phase 5 Gateway}

In this section, the development of a phase 5 Gateway to be used in IoT is presented. As described in 2.2, phase 5 Gateway can be installed in outside plants for various IoT services. In this section, it is illustrated that the phase 5 is applied to the wildlife monitoring service as one of smart city applications. A summary of a wildlife monitoring system in the FED4IoT project is discussed. In addition, development of the Gateway 
based on the requirements discussed in Section 3 and its application to the wildlife monitoring system are presented.

\subsection{Summary of the Smart City Application}

With an increase in the IoT, smart-city applications are often discussed. Home security [9], smart factory [10], smart building [11] are typical examples of IoT applications. A smart city application is an application in which diverse IoT systems are interoperated. The FED4IoT project, which we join, aims to develop a similar smart-city application [12], [13]. A schematic of the FED4IoT project is shown in Figure 5. As shown in this figure, the FED4IoT has four locations and five applications are provided in each location. An interoperation of the IoT data is attempted. In this project, smart-city applications are developed in the cloud servers, where the data interoperation also takes place. Therefore, as an entrance to the FED4IoT cloud system, using a cloud server is required for data management on the cloud. In this project, FIWARE [14] and oneM2M [15] were chosen for data management on the cloud.

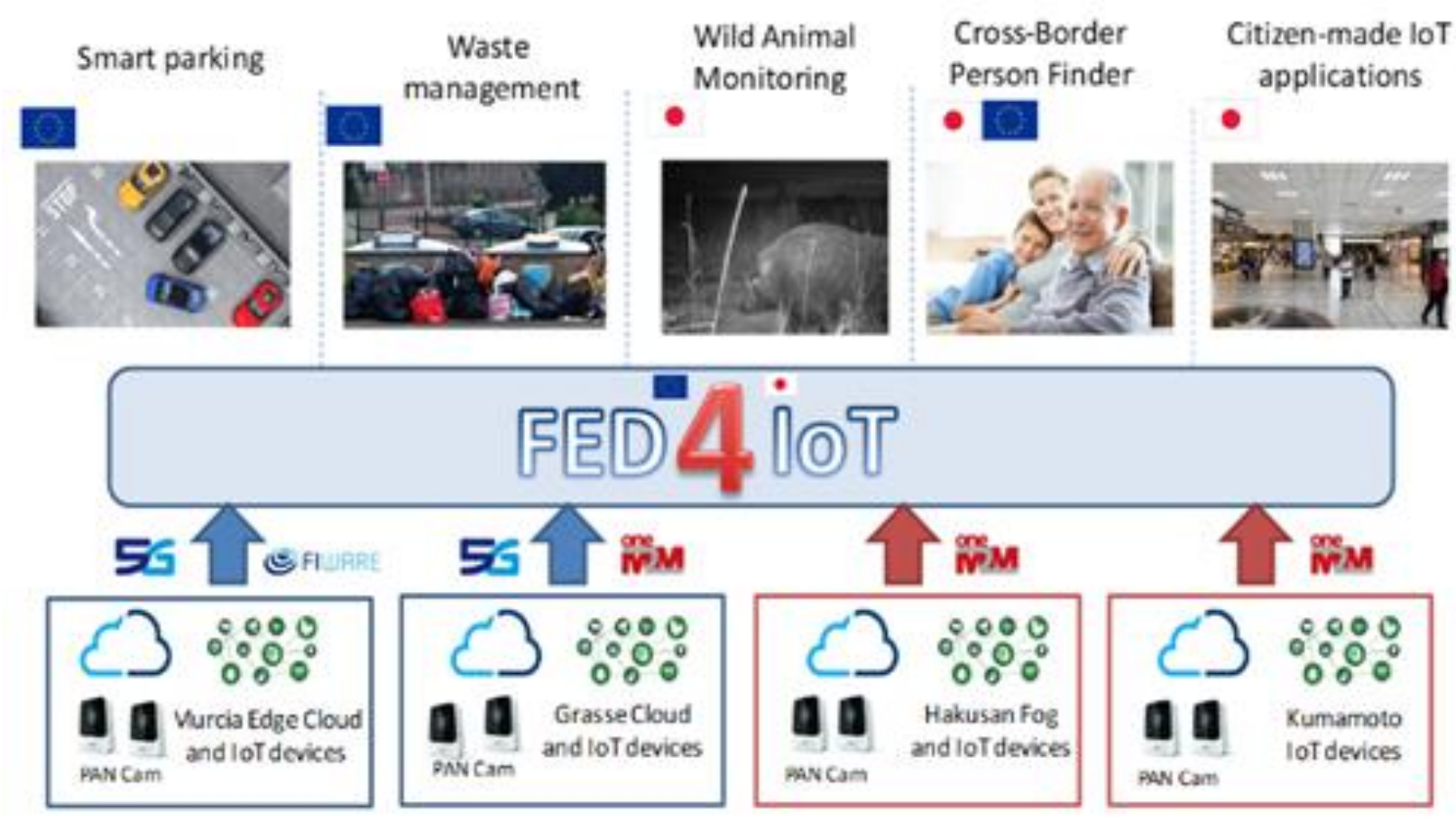

Fig. 5: Schema of the FED4IoT

\subsection{Wildlife Monitoring System using the IoT and Verification Model}

In the FED4IoT project, Kanazawa Institute of Technology chose a wildlife monitoring system as a use case of IoT applications. Its architecture, including the phase 5 Gateway, is shown in Figure 6. Further information on this wildlife monitoring system is provided in Reference [16].

In this figure, three patterns of the end devices are provided. Pattern 1 consists of a camera monitoring the animal approaching the residential areas; Pattern 2 consists of cameras and cages with sensors, and Pattern 3 for remote areas consisting of cages with sensors. Data from each end device are integrated at the Gateway, and data processing (analysis and format conversion) takes place. Finally, the converted data are posted on a cloud server. In this system, FIWARE was chosen as an example of cloud services.

In this study, the gateway, which was applied to Pattern 3 end device in Figure 6, was developed. The verification model of this study is shown in Figure 7. Pattern 3 consists of environmental sensors (temperature, humidity, illuminance, and raindrop) and a pressure sensor monitoring the cage's condition. Low-power wide area networking technology (LoRaWAN) was chosen for communication between the phase 5 Gateway and sensors. As shown in the figure, data from the sensors were collected at the LoRa Mini device and then transferred to the LoRa Gateway. The Gateway receives the data through the secure shell (SSH). The phase 5 Gateway analyzes the received data and converts it into a FIWARE determined format. Finally, the converted data are posted to the FIWARE server. 


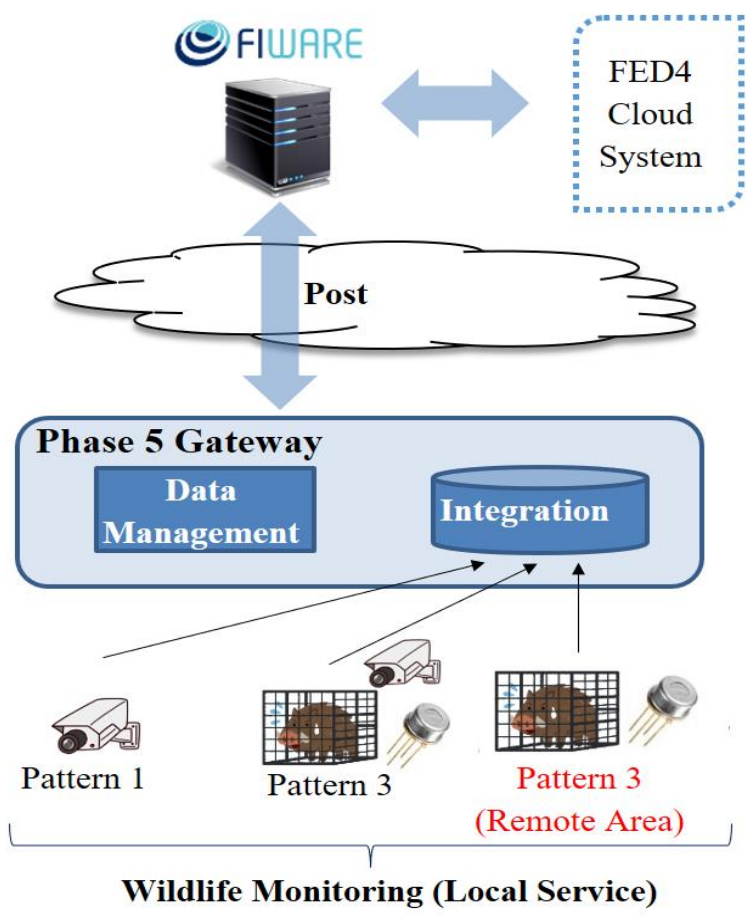

Fig. 6: Structure of phase 5 Gateway applied to the wildlife monitoring system

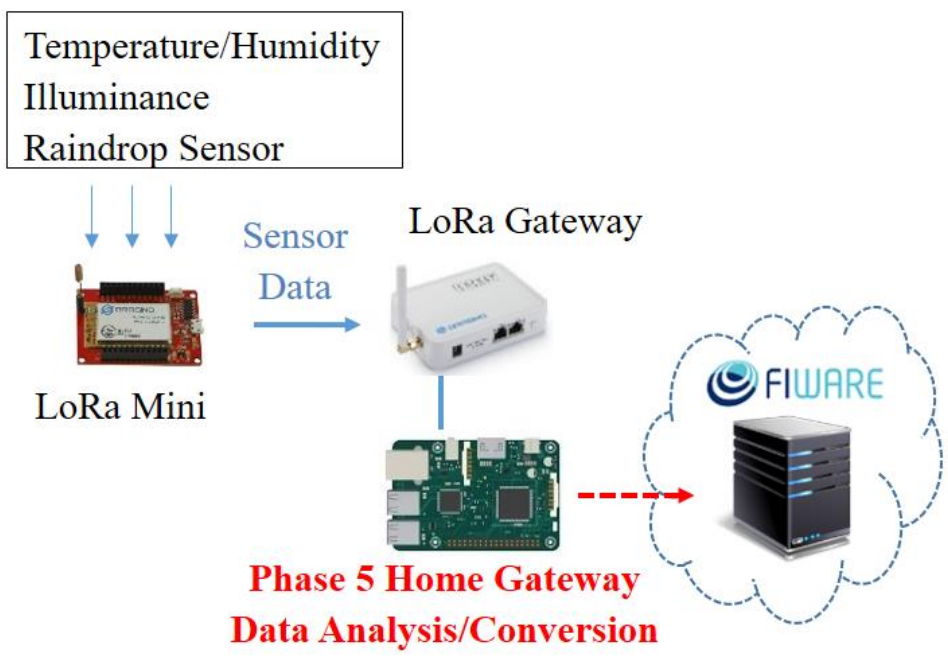

Fig. 7: Verification model of Pattern 3 end device

\subsection{Specification of the Data Model}

To use FIWARE as a cloud service, the gateway needs to support its data format. Moreover, it is necessary to know the type of data obtained from the wildlife monitoring at the gateway. Therefore, a data model of the wildlife monitoring system was created. The data model for Pattern 3 and the common data are shown in Table 1. The data models were created based on the FIWARE, which is an entity-attribute type data model [17]. The entity contains attributes such as the sensor data and time data. Common data are a dataset for the whole wildlife monitoring system. Under these attributes, dateissued is the exact time when the common data are received; Type is an attribute's format, such as a number or letter; Unit refers to the unit, such as degree or lx. 
Table. 1: Data model of the pattern 3 and the common data

\begin{tabular}{|c|c|}
\hline Entity & Attributes \\
\hline \multirow{5}{*}{ CommonData } & $\begin{array}{ll}- & \text { Name : dataissued } \\
- & \text { Type } \\
- & \text { Value }\end{array}$ \\
\hline & $\begin{array}{ll}\text { - } & \text { Name : temperature } \\
\text { - } & \text { Type } \\
\text { - } & \text { Value } \\
\text { - } & \text { Unit } \\
\end{array}$ \\
\hline & $\begin{array}{ll}- & \text { Name : humidity } \\
\text { - } & \text { Type : } \\
\text { - } & \text { Value } \\
\text { - } & \text { Unit }\end{array}$ \\
\hline & $\begin{array}{ll}\text { - } & \text { Name : illuminance } \\
\text { - } & \text { Type } \\
\text { - } & \text { Value } \\
\text { - } & \text { Unit } \\
\end{array}$ \\
\hline & $\begin{array}{ll}\text { - } & \text { Name : raindrop } \\
\text { - } & \text { Type } \\
\text { - } & \text { Value } \\
\text { - } & \text { Unit } \\
\end{array}$ \\
\hline \multirow[b]{2}{*}{ Patter3 } & $\begin{array}{ll}- & \text { Name : dateissued } \\
- & \text { Type } \\
- & \text { Value } \\
\end{array}$ \\
\hline & $\begin{array}{ll}- & \text { Name : CageStatus } \\
- & \text { Type } \\
- & \text { Value } \\
- & \text { SensorId }\end{array}$ \\
\hline
\end{tabular}

\subsection{Development of Phase 5 Gateway Applied to Wildlife Monitoring System}

Figure 8 shows the results of the collected sensor data, which were observed with serial communication at the LoRaWAN Gateway. In this figure, "DataID:101" is an ID of the cage data of Pattern 3 and includes the data of the pressure sensor. If the cage physically touches the pressure sensor, then integer " 1 " will be sent to the LoRaWAN Gateway. If the cage is open and is not physically touched by the pressure sensor, then integer " 0 " will be sent to the LoRaWAN Gateway. The data with "DataID: 001" have common data. Common data include the temperature, humidity, illuminance, and raindrop data. The received data at the LoRaWAN gateway are transferred to the RDG through a secure shell communication.

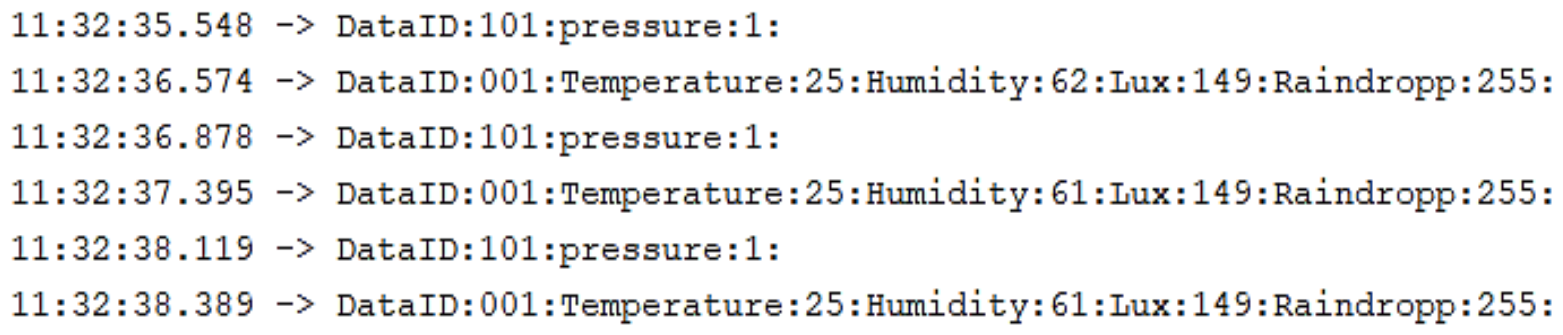

Fig. 8: Sensor data received at the LoRaWAN Gateway

Figure 9 shows the output at the terminal of phase 5 Gateway. As shown in this figure, the phase 5 Gateway receives the common data and the data of pattern 3 from the LoRaWAN Gateway, analyzes them, and converts their data formats into FIWARE determined format. The top in the output is a dataset of Pattern 
3 end device, and the bottom is a dataset of the common data. Phase 5 gateway recognizes the data as the common data or the data from Pattern 3 through the "DataID." The condition of the cage in Pattern 3 is recognized with the data of the pressure sensor. Because the pressure sensor was "1," as shown in Figure 8, phase 5 Gateway recognized that the animal was in the cage, as shown at the top of Figure 9.

" $<$ Response [204] $>$ " is a response sent by the FIWARE server, which means that the data were updated successfully. Even though a time lag occurred between the LoRaWAN Gateway and phase 5 Gateway, the phase 5 Gateway succeeded in analyzing the IoT data, transforming its data format, and posting the data into the FIWARE server.

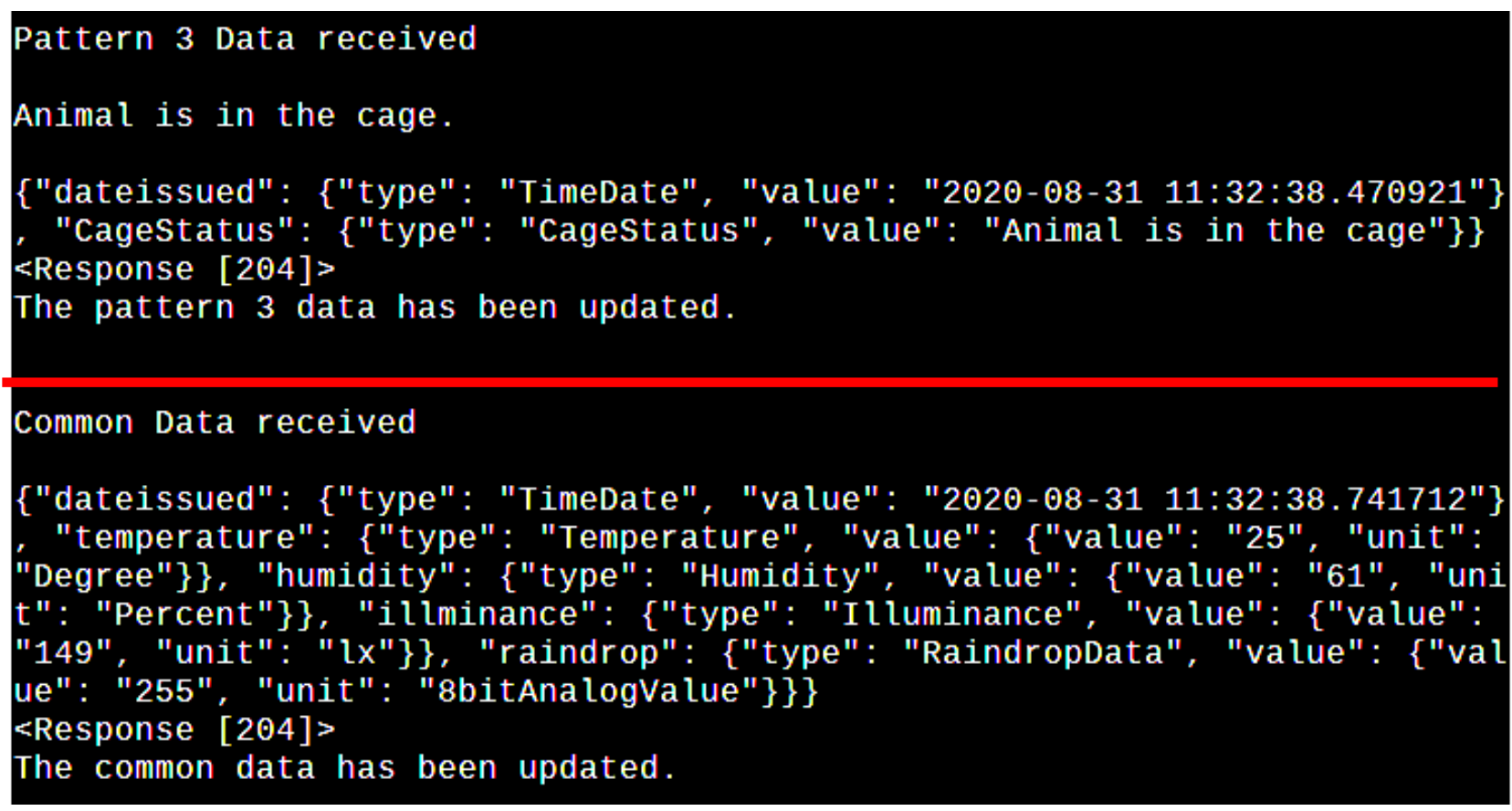

Fig. 9: Analyzed and transformed data at the RDG

\section{Conclusion}

With the introduction of IoT over the $5 \mathrm{G}$ mobile system, internet services have become more diverse. Therefore, the requirements of the gateway have changed. In this study, the transition of the home gateway based on four phases has been discussed, and the requirements of the next phase of the Gateway applied to IoT services are presented. The phase 5 Gateway needs to possess open connectivity, capability of cooperation with the cloud services, and capacity of IoT sensors and other devices through the home network (local network). The FED4IoT project was presented as a use case of the development of phase 5 Gateway, and a summary of the wildlife monitoring system was discussed. The development of Phase 5 home gateway, which is based on the presented requirements and applied to the wildlife monitoring system, has been presented. Improvements in the Gateway to be applied to the left end devices, Pattern 1 and Pattern 2, have been planned for the future.

\section{Acknowledgment}

The research leading to these results has been supported by the EU-JAPAN initiative through the EC Horizon 2020 Work Programme (2018-2020) Grant Agreement No. 814918 and Ministry of Internal Affairs and Communications' program of "federating IoT and cloud infrastructures to provide scalable and interoperable Smart City applications, by introducing novel IoT virtualization technologies (Fed4IoT)."

\section{References}

[1] Sukegawa, V. Tomohiro Ishihara V. Kiyoshi, and V. Hirokazu Shimada. "Home Gateway enabling evolution of network services." FUJITSU Sci. Tech. J 42.4 (2006): 446-453.

[2] Kramer, Glen. What is Passive Optical Network? Vol. 17. McGraw-Hill, 2005. 
[3] Oh, JongGyu, et al. "A study of development of transmission systems for next-generation terrestrial 4K UHD \& HD convergence broadcasting." Journal of Broadcast Engineering 19.6 (2014): 767-788.

[4] Dorsemaine, Bruno, et al. "Internet of things: a definition \& taxonomy." 2015 9th International Conference on Next Generation Mobile Applications, Services and Technologies. IEEE, 2015.

[5] Fukuyama, Mayumi. "Society 5.0: Aiming for a new human-centered society." Japan Spotlight 27 (2018): 47-50.

[6] Lasi, Heiner, et al. "Industry 4.0." Business \& information systems engineering 6.4 (2014): 239-242.

[7] Kenichi Ooto, Tetsuya Hirano, Kota Hidaka, Toshinori Kikuchi, “Applying home ICT for developing home network services”, IEICE Technical Report, Vol.111, No.118, ICM2011-9, Jun., 2011

[8] OSGi Alliance, accessed on September 14, 2020 [Online] Available https://www.osgi.org/

[9] Kodali, Ravi Kishore, et al. "IoT based smart security and home automation system." 2016 international conference on computing, communication and automation (ICCCA). IEEE, 2016.

[10] Wang, Shiyong, et al. "Implementing smart factory of industrie 4.0: an outlook." International journal of distributed sensor networks 12.1 (2016): 3159805.

[11] Plageras, Andreas P., et al. "Efficient IoT-based sensor BIG Data collection-processing and analysis in smart buildings." Future Generation Computer Systems 82 (2018): 349-357.

[12] Ogawa, Keigo, et al. "IoT Device Virtualization for Efficient Resource Utilization in Smart City IoT Platform." 2019 IEEE International Conference on Pervasive Computing and Communications Workshops (PerCom Workshops). IEEE, 2019.

[13] Hidenori Nakazato, "IoT Networks and Their Federation", JAC-ECC 2018, IEICE Invited session \#2, 2018.

[14] Fazio, Maria, et al. "Exploiting the FIWARE cloud platform to develop a remote patient monitoring system." 2015 IEEE Symposium on Computers and Communication (ISCC). IEEE, 2015.

[15] Alaya, Mahdi Ben, et al. "Toward semantic interoperability in oneM2M architecture." IEEE Communications Magazine 53.12 (2015): 35-41.

[16] Yoshida, Eisei, et al. "Concept for and Implementation of Wildlife Monitoring to Contribute Sustainable Development Goals." 2019 International Conference on Innovation and Intelligence for Informatics, Computing, and Technologies (3ICT). IEEE, 2019.

[17] How to - FIWARE - Data Models, accessed on August 8, 2020 [Online] Available https://fiwaredatamodels.readthedocs.io/en/latest/howto/index.html 